\title{
Voice Characteristics of People with Parkinson's Disease (PwP): An Overview on Baseline Vocal Characteristics and Guidelines for Therapeutic Singing
}

\author{
Ang Mei Foong \\ Department of Music, Faculty of Human Ecology, Universiti Putra Malaysia \\ 43400 Serdang, Selangor, Malaysia \\ e-mail: meifoong@upm.edu.my
}

Published online: 28 December 2018

Cite this article (APA): Ang, M.F. (2018). Voice characteristics of people with Parkinson's disease $(\mathrm{PwP})$ : An overview on baseline vocal characteristics and guidelines for therapeutic singing. Malaysian Journal of Music, 7, 208-224.

\begin{abstract}
Parkinson's Disease (PD) is a chronic neurodegenerative disease in which the symptoms of the disease are worsen over time. Core symptoms of the disease are such as tremor-at-rest, rigidity, postural instability, and bradykinesia. Apart from these motor-problems, other problems such as sleep disorder, sensory abnormalities, neuropsychiatric disorders also exist. In addition to the motor-problems, latest literature documented $80-90 \%$ of the people with Parkinson's Disease (PwP) are prone to develop voice impairment known as Parkinsonian Dysarthria, when symptoms deteriorate, causing loss of communication, difficulties in swallowing and breathing that are psychologically devastating and life threatening. Growing research in music therapy (MT) and music-based rehabilitative activities worldwide documented positive effects on PwP. A local research using therapeutic singing as rehabilitative model at the Malaysian Parkinson's Disease Association (MPDA) also have reported positive benefits in improving vocal problems in the Malaysian PwP. In this research, some primary and preliminary data on the vocal characteristic, including baseline vocal loudness (VL), pitch range (PR) and respiratory rate (RR) were obtained among 22 local PwP prior to examining effects of singing on PwP. Findings of this study showed that the PwP speak softer than the normal accepted range. The findings also showed that they have tendency to develop rapid breathing, and their average pitch range while singing is limited within the range of G\#3 - B4. These findings can further propose guidelines for voice rehabilitation models based on the preliminary voice characteristics concluded in this study.
\end{abstract}

Keywords: Parkinson's disease, Parkinsonian Dysarthria,voice characteristics, therapeutic singing, 


\section{Introduction}

Parkinson's Disease (PD) is a neurodegenerative disease that is caused by reduction in the dopamine (that serves as a neurotransmitter in the nervous-system) in which the symptoms of the disease deteriorate over time (Lim, Puvanarajah, Lee, Chee, Viswanathan, Tan, Chong \& Azrin, 2012; Schulz \& Grant, 2000). PD causes both motor and non-motor symptoms, and it has a tendency to provoke mental health issues (Aarsland, Larsen, Karlsen, Lim, \& Tandberg, 1999; Branchi, D'Andrea, Armida, Cassano, Pezzola, Potenza, Morgese, Popoli, \& Alleva, 2008; Jankovic, 2008; Meireles \& Massano, 2012; Rutten, Ghielen, Vriend, Hoogendoorn, Berendse, Leentjens, van der Werf, Smit, \& van den Heuvel, 2014).

There are four core symptoms in PD: tremor at rest, rigidity, bradykinesia (slowness), and postural instability. However, more of other motor-symptoms, such as freezing and dyskinesia also observed among PwP at different stages of the disease (Dyduch \& Załuska, 2014; Jankovic, 2008; Lim, Fox, \& Lang, 2009; Politis, Wu, Mollis, Bain, Chaudhuri \& Piccini, 2010). The non-motor symptoms are such as constipation, pain, sleep disorders, cognitive impairments, poor memory, slowness in psychological responses, problems of visuospatial functioning (Aarsland, Larsen, Karlsen, Lim, \& Tandberg, 1999; Jenkinson, Peto, Fitzpatrick, Greenhall, \& Hyman, 1995; Meireles \& Massano, 2012; Pagonabarraga, Kulisevsky, Strafella, \& Krack, 2015), and sensory motor integration impairments (Clark, Adams, Dykstra, Moodie, \& Jog, 2014; Jankovic, 2008; Ramig, Fox, \& Sapir, 2007; Richardson, Sussman, Stathopoulos, \& Huber, 2014). These symptoms could emerge before the manifestation of the motor problems (Lim et al., 2009). In addition to that, PwP frequently suffers from neuropsychiatric symptoms, such as depression (Branchi et al., 2008), anxiety (Rutten et al., 2014), apathy (Pagonabarraga et al., 2015), hallucination/delusions (Meireles \& Massano, 2012) and psychosis (Aarsland et al., 1999).

\section{Speech Impairments in Parkinson's Disease}

Among the motor-problems the PwP are facing, voice impairment in PD is one of the most prominent symptoms. Studies pointed out that $\mathrm{PwP}$ are prone to develop hypokinetic dysarthria (HD, also known as Parkinsonian dysarthria), a voice and speech production problem due to PD (Miller, Noble, Jones, \& Burn, 2006; Ramig et al., 2007). Some early studies in the 1980s suggested that there are 50\% of PwP presenting communication difficulties (Scott \& Caird, 1983). Later studies in 2006 proposed that the percentage of $\mathrm{PwP}$ who showed impairments in speech is close to 80\% (Miller et al., 2006); and more recent studies by Ramig and Fox predicted that there are near to $90 \%$ of the PwP demonstrating deficits in speech (Ramig et al., 2007), which has raised an alarming alert to this problem.

HD happens when movements of the larynx reduced due to inadequate muscle activation. Typical symptoms in HD include softness in speech (hypophonia), monotone (dysprosody), imprecise articulation (slurred in speech), vocal tremor, and quality of voice becoming hoarse and breathy; on the other hand, the inactive movements in the lungs and respiratory systems resulted in reduced 
breath supports (Buetow, Talmage, McCann, Fogg, \& Purdy, 2014; Elefant, Baker, Lotan, Lagesen, \& Skeie, 2012; Fogg-Rogers, Buetow, Talmage, McCann, Leao, Tippett, Leung, McPherson, \& Purdy, 2015; Haneishi, 2001; Ramig et al., 2007; Schulz \& Grant, 2000).

$\mathrm{HD}$ is generally regarded as part of the natural deterioration of $\mathrm{PD}$, however, in addition to the natural deterioration of the disease, it was also reported that PwP who had undergone Deep Brain Surgery (STN-DBS, deep brain surgery of the subthalamic nucleus) has higher tendency to manifest voice impairments (Wertheimer, Gottuso, Nuno, Walton, Duboille, Tuchman, \& Ramig, 2014). Another study filed report that speech impairments in PD could be an adverse drug event to Dopamine Agonist (DA), which is a common pharmacological treatment for PwP (Cacciatore, Simone, Patrocinio, \& Sallustio, 2012).

There are a few major impacts on the PwP and their caregivers due to the changes in their vocal quality and speech intelligibility in their daily lives. A qualitative study identified and characterized four main impacts due to speech impairments from the PwP' perspectives: 1) interaction with others; 2) problems with conversations; 3) feelings about intelligibility; and 4) the voice. The main concern among the PwP was not merely about the change of voice itself but the poor communication that resulted in loss of dignity (Miller et al., 2006).

The impaired voice and speech doesn't only affect the PwP but it also creates serious impact on the PwP' caretakers and their immediate family members, as it causes frustrations and further leading the PwP to withdraw from the daily conversations as well as social events, which then increase the risks of developing psychological disorders such as depression and anxiety which seriously affect their quality of life (Aarsland et al., 1999; Miller, Deane, Jones, Noble, \& Gibb, 2011; Miller et al., 2006; Ramig \& Fox, 2007).

\section{Treatments in Parkinsonian Dysarthria}

The present treatments for PD are mainly pharmacological. Medications such as levodopa (L-dopa) is often used to treat motor symptoms in PD. L-dopa is used to restore dopamine level in the brain, where the reduction of which is the cause of the disease. Common levodopa-containing drugs are such as Madopar ${ }^{\circledR}$ and Sinemet@ (for initial state of PD); other medications such as selegiline, amantadine, dopamineagonists can also be prescribed (Lim et al., 2012). For treatments related to speech impairments in PD, there are studies which have reported unsatisfactory outcomes from both pharmacological and surgical treatments (Pinto, Ozsancak, Tripoliti, Thobois, Limousin-Dowsey, \& Auzou, 2004; Schulz, Greer, \& Friedman, 2000). Both treatments filed speech deficits in PwP following STN-DBS (Wertheimer et al., 2014) or reported as an adverse drug event following DA (Cacciatore, Simone, Patrocinio, \& Sallustio, 2012). Even though both treatments reported significant improvements to the limbs' motor functions, however deficits in speech intelligibility were reported, the studies thus concluded that improvements in limbs do not associate with improvements in voice. 
In contrary to pharmacological and surgical treatment, behavioral treatments such as speech therapy and music therapy that focus on conscious and repeated training to strengthen vocal muscles appears to be a more effective way to treat HD (Atkinson-Clement, Sadat, \& Pinto, 2015; Pinto et al., 2004; Schulz \& Grant, 2000). Among speech therapies, the Lee Silvermann Voice Treatment ${ }^{\circledR}$ (LSVT) has proven over these two decades to have received positive results in treating speech impairments in PwP. Vast literatures and meta analyses documented the effects of LSVT in treating HD among PwP and majority of which reported positive outcomes in the parameters of vocal loudness, phonatory, articulation, and respiration. The classic LSVT is conducted four times per week within 4 weeks and it was claimed that the effects could last for two years after treatment (Ramig \& Fox, 2007). On the other hand, growing number of research in MT, including MT that focus on singing as intervention, also demonstrated positive effects in improving motor functions, speech impairments, facial expressions, and mood among PwP (Elefant, Lotan, Baker, \& Skeie, 2012; Elefant et al., 2012; Evans, Canavan, Foy, Langford, \& Proctor, 2012; Haneishi, 2001; Pacchetti, Mancini, Aglieri, Fundarò, Martignoni, \& Nappi, 2000). More specifically, a few studies in MT have shown singing techniques to have helped to extent chest capacity, improves respiratory muscles movements and hence helps to improve breathing, leading to significant improvements in voice productions (Evans, Canavan, Foy, Langford, \& Proctor, 2012; Tanday, 2016).

Another small study that examines efficacy of Music Therapy Voice Protocol (MTVP) on speech intelligibility (how speech is comprehensible) and vocal intensity revealed some small but positive improvements in the acoustic variables which include speech intelligibility, vocal intensity, maximum vocal range, maximum duration of sustained vowel phonation, vocal fundamental frequency, vocal fundamental frequency variability and mood. MTVP runs for three times weekly for a duration of 12-14 weeks. Even though some parameters didn't achieve statistical significance due to small sample size $(\mathrm{N}=4)$, however improvements in means were detected and it was suggested that singing may influence vocal projections leading to improvements in speech (Haneishi, 2001).

Other similar studies were carried out thereafter. A study done by Elefant et al. in 2012 also reported significant positive effects in singing quality and vocal range but reported no changes to quality of speech. The intervention was based on group singing, participants received 60-minutes singing intervention in small group setting for 20 consecutive weeks. There were $10 \mathrm{PwP}$ who are stable responders to levodopa and in Hoehn and Yahr scale (stage 2 or 3) participated in this one-group repeated measure design study. Research result showed significant improvements in five of the six singing qualities (fluency, singing accuracy, intensity of $\mathrm{dB}$, consistency of $\mathrm{dB}$, voiceless, voiced) at Time 2 and $3(\mathrm{p}<.01)$, as well as voice range and the Vocal Handicap Index (VHI) physical subscale at Time 3 (Elefant et al., 2012).

In addition to the above studies, another two-years longitudinal study in UK reported small but significant improvements in laryngeal elements and small degree of improvements in participants' self-rating quality of life, suggesting that group singing helps to prevent deterioration in voice and to maintain voice quality among 
the PwP (Evans et al., 2012). Another study of PwP' experience in Choral Singing Therapy (CST) in New Zealand reported that choral singing helped PwP to selfmanage some consequences of their conditions, which include low mood, social isolation and communication difficulties (Fogg-Rogers et al., 2015).

\section{Voice Characteristics}

Even though growing researches have been focused on testing the efficacy of musicbased therapeutic protocols on voice impairments in the PwP, but limited research had reported the voice characteristics of the PwP. These include finding out the baseline in vocal loudness, pitch ranges and respiratory rates in the PwP. These baseline data are important to allow voice-rehab instructors or music therapists to draft a more reasonable rehabilitation plan that is feasible and possible for the PwP to master, in order to achieve the overall rehabilitation purposes and to avoid any unforeseeable harm to the voice due to a wrong choice of repertoire. A good decision on the repertoire takes account of the baseline ability of the PwP and a good set of repertoires will be able to achieve rehabilitation objectives without probability to harm the voice.

\section{Methodology and Procedures}

A total of 22 PwP from the Malaysian Parkinson's Disease Association participated in a research that study effects of a singing protocol on PwP. These PwP consisted of 10 males $(45.5 \%)$ and 12 females $(54.5 \%)$ ranging in age from 45 to 86 years old $(\mathrm{M}=68.4 \mathrm{y} / \mathrm{o})$, with 45 years old (y/o) being the youngest participant and $86 \mathrm{y} / \mathrm{o}$ being the oldest participant, with a mean age at 68.41 y/o $(\mathrm{SD}=8.921)$. The research participants had lived with $\mathrm{PD}$ for a mean time of $8.7(\mathrm{SD}=5.715)$ years, ranging from 1 year to 20 years ( $M=8.77$ years).

The participants in this study were first measured their baseline vocal parameters before following through a series of voice rehabilitation activities. The vocal parameters in this study focused on better understanding the vocal problems/ symptoms following HD, hypophonia (vocal loudness), issues related with breathing, and dysprosody (monotonous, lack of pitch range in speech). Therefore, the baseline vocal parameters that were measured in this study included both the minimum amplitude (softest voice) at read and maximum amplitude (loudest voice) at read; respiratory rate; as well as both minimum pitch (lowest note the PwP can reach) and maximum pitch (highest note the PwP can reach). Each procedure will be further elaborated in the description below:

Vocal Amplitude. Both min amplitude and max amplitude data were obtained when participants were reading a passage at their normal comfortable volume, mimicking a closest possible situation to normal daily speech. The sound meter was placed at the distance of $8 \mathrm{~cm}$ from the participants' mouth, data were noted in decibels $(\mathrm{dB})$.

The data for vocal amplitude was obtained through a smartphone app (see Figure 1) that functions as a sound pressure level (SPL) meter called Sound Meter V.1.6.13 developed by Smart tools co. Both min and max score (softest and loudest) 
of sound level could be detected during a same measurement time. Figure 1 shows a sample of data, it can be clearly seen that the min score (softest voice) and max score (loudest voice) in a reading session were obtained, in which the $\min =62 \mathrm{~dB}$ and $\max =79 \mathrm{~dB}$.

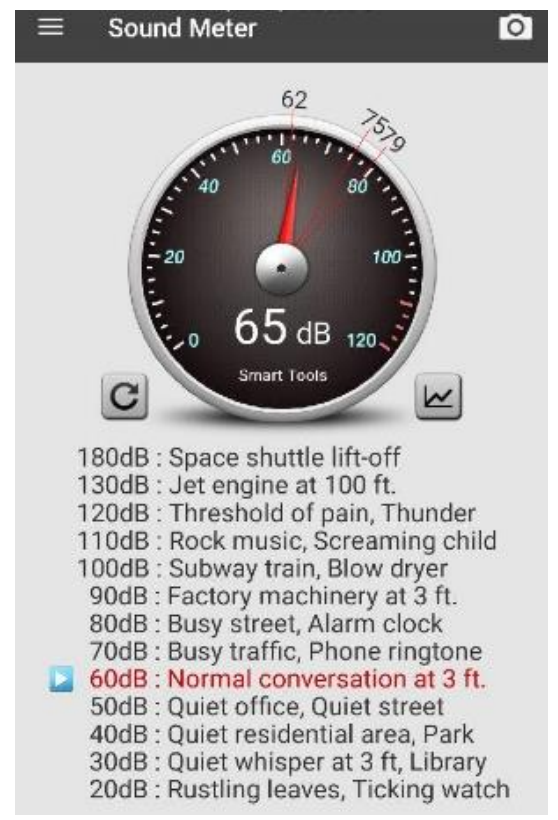

Figure 1. An example of acoustic data through 'Sound Meter' app.

Respiratory Rate. Respiratory rate refers to the number of breaths per minute. Healthy adults' respiratory rate is ranged between 12-20. In the context of this study, Respiratory Rate in PwP is measured with smartphone app named iCare Respiratory Rate V.3.6.0 (see Figure 2) in the unit of breath-per-minute. Respiratory rate data was obtained when the participants are at relaxed sitting posture before a singing activity takes place. Figure 2 shows the overview of the smartphone app. 


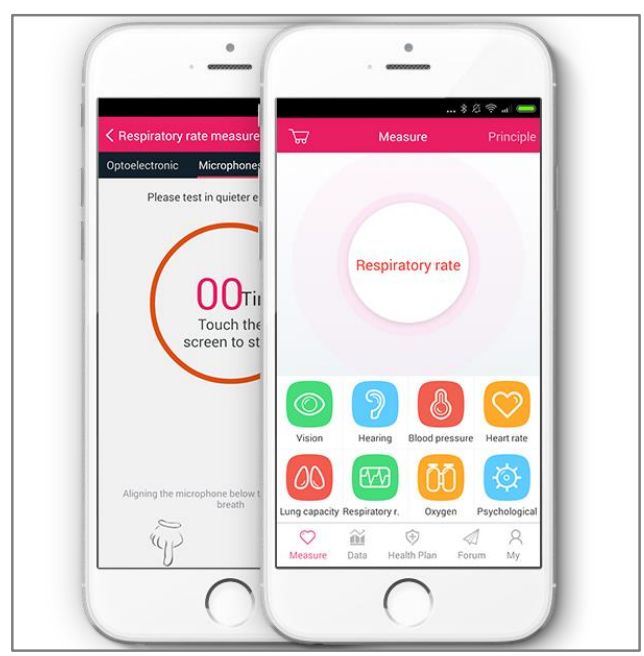

Figure 2. iCare respiratory rate app.

Pitch Range. Pitch range was measured individually to determine the lowest and the highest pitch a participant could reach. PwP tend to speak in monotone with a decreased pitch range thus affecting the quality of their speech, hence it is important to understand the issues by grasping their baseline pitch ranges and to further drafting rehabilitation plan to expand their pitch range. Participants were asked to sing a set of researcher's created vocalises (see Figure 3, Figure 4, Figure 5, and Figure 6) and each vocalise was played on a music keyboard to accompany participants and assessed by the researcher. Both the highest (max) and lowest (min) pitches were recorded according to musical notation and then translated to frequency $(\mathrm{Hz})$ when analyzing data.

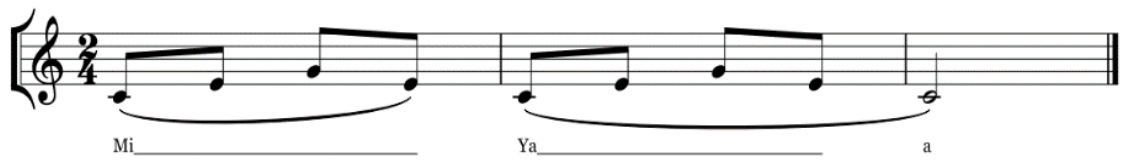

Figure 3. Vocalise Type A.

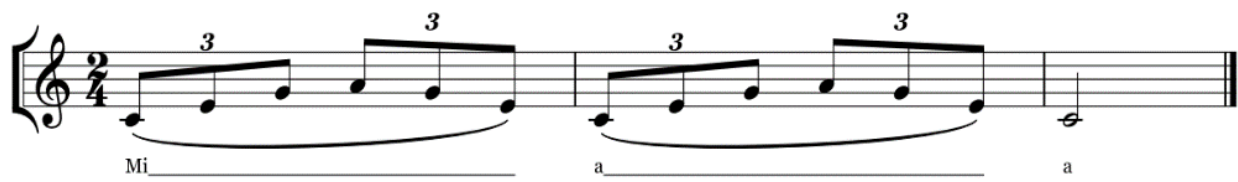

Figure 4. Vocalise Type B.

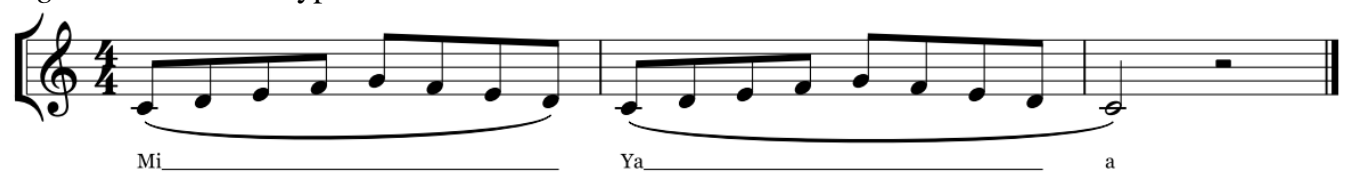

Figure 5. Vocalise Type C 


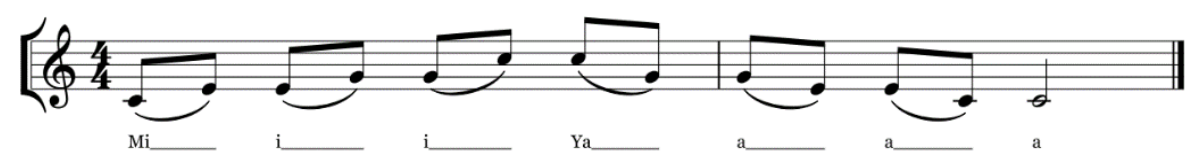

Figure 6. Vocalise Type D.

\section{Results}

\section{Vocal Amplitude}

Table 1 below shows the descriptive statistics of the min vocal amplitude at read. Lowest min vocal amplitude score was noted at $45 \mathrm{~dB}$, whilst highest min vocal amplitude score was noted at $58 \mathrm{~dB}$, with its mean score $=52.09 \mathrm{~dB}$ among the 22 PwP participants.

Table 1

Descriptive Statistics of Min Vocal Amplitude at Read

\begin{tabular}{llcccc}
\hline & $\mathrm{N}$ & Minimum & Maximum & Mean & Std. Deviation \\
\hline AMP read min & 22 & 45.00 & 58.00 & 52.0909 & 3.71495 \\
\hline Valid N (listwise) & 22 & & & & \\
\hline
\end{tabular}

Table 2 below listed out the frequencies of min vocal amplitude by number of participants. Highest frequencies of min vocal amplitude were noted at $52 \mathrm{~dB}$ (4 participants) which is very close to the mean min vocal amplitude score.

Table 2

Frequencies of Min Vocal Amplitude by Number of Participants

\begin{tabular}{cccccc}
\hline & Frequency & Percent & Valid Percent & Cumulative Percent \\
\hline Valid & 1 & 4.5 & 4.5 & 4.5 \\
\cline { 2 - 6 } & 45.00 & 1 & 4.5 & 4.5 & 9.1 \\
\cline { 2 - 6 } 46.00 & 1 & 4.5 & 4.5 & 13.6 \\
\hline 477.00 & 1 & 4.5 & 4.5 & 18.2 \\
\hline 48.00 & 1 & 13.6 & 13.6 & 31.8 \\
\hline 49.00 & 4 & 4.5 & 4.5 & 36.4 \\
\hline 51.00 & 1 & 18.2 & 18.2 & 54.5 \\
\hline 52.00 & 4.5 & 4.5 & 59.1 \\
\hline
\end{tabular}




\begin{tabular}{ccccc}
\hline 54.00 & 2 & 9.1 & 9.1 & 68.2 \\
\hline 55.00 & 2 & 9.1 & 9.1 & 77.3 \\
\hline 56.00 & 3 & 13.6 & 13.6 & 90.9 \\
\hline 57.00 & 1 & 4.5 & 4.5 & 95.5 \\
\hline 58.00 & 1 & 4.5 & 4.5 & 100.0 \\
\hline Total & 22 & 100.0 & 100.0 & \\
\hline
\end{tabular}

Similarly, Table 3 below shows the descriptive statistics of max vocal amplitude at read. Lowest max vocal amplitude noted was $65 \mathrm{~dB}$, whilst highest max vocal amplitude was noted at $86 \mathrm{~dB}$, with its mean score $=74.68 \mathrm{~dB}$ among the 22 PwP participants.

Table 3

Descriptive Statistics of Max Vocal Amplitude at Read

\begin{tabular}{lccccc}
\hline & N & Minimum & Maximum & Mean & Std. Deviation \\
\hline AMP read max & 22 & 65.00 & 86.00 & 74.6818 & 4.83427 \\
\hline Valid N (listwise) & 22 & & & & \\
\hline
\end{tabular}

Table 4 below shows frequencies of max vocal amplitude by number of participants. Highest frequencies of max vocal amplitude were noted at $75 \mathrm{~dB}$ (4 participants), which is also very close to the mean max vocal amplitude score.

Table 4

Frequencies of Max Vocal Amplitude by Number of Participants

\begin{tabular}{cccccc}
\hline & Frequency & Percent & Valid Percent & Cumulative Percent \\
\hline Valid & 1 & 4.5 & 4.5 & 4.5 \\
\cline { 2 - 6 } & 65.00 & 1 & 4.5 & 4.5 & 9.1 \\
\hline & 66.00 & 2 & 9.1 & 9.1 & 18.2 \\
\hline 70.00 & 2 & 9.1 & 9.1 & 27.3 \\
\hline 72.00 & 2 & 9.1 & 9.1 & 36.4 \\
\hline 73.00 & 2 & 9.1 & 9.1 & 45.5 \\
\hline 74.00 & 4 & 18.2 & 18.2 & 63.6 \\
\hline
\end{tabular}




\begin{tabular}{ccccc}
\hline 76.00 & 3 & 13.6 & 13.6 & 77.3 \\
\hline 78.00 & 1 & 4.5 & 4.5 & 81.8 \\
\hline 79.00 & 1 & 4.5 & 4.5 & 86.4 \\
\hline 81.00 & 1 & 4.5 & 4.5 & 90.9 \\
\hline 82.00 & 1 & 4.5 & 4.5 & 95.5 \\
\hline 86.00 & 1 & 4.5 & 4.5 & 100.0 \\
\hline Total & 22 & 100.0 & 100.0 & \\
\hline
\end{tabular}

\section{Respiratory Rate}

For respiratory rate, it is important to note that the healthy respiratory rate in a healthy adult should be within the range of 12 to 20 breaths per minute. Table 5 below showed descriptive statistics of the scores of the respiratory rate with min score $=14$ breaths $/$ min and max score $=28$ breaths $/$ min, with its mean score $=20$ breaths/min. The mean score shows that the respiratory rate was within the healthy range however it was at the border of the higher side signifying a risk to rapid breathing.

Table 5

Descriptive Statistics of Respiratory Rate

\begin{tabular}{lccccc}
\hline & N & Minimum & Maximum & Mean & Std. Deviation \\
\hline RESPIRATORY R & 22 & 14.00 & 28.00 & 20.0000 & 3.08607 \\
\hline Valid N (listwise) & 22 & & & & \\
\hline
\end{tabular}

To understand this scenario better, frequencies of respiratory rate by number of participants were listed out, as shown in the Table 6 below. The table displayed that 13 participants' respiratory rate was within the healthy range of 12 to 20 breaths/min, whilst 9 participants' respiratory rate had gone above 20 breaths $/ \mathrm{min}$, with the highest frequencies noted at 19 breaths/min and 22 breaths/min (4 participants each). This has raised some alarming alert in issues related with breathing among the PwP. 
Table 6

Frequencies of Respiratory Rate by Number of Participants

\begin{tabular}{cccccc}
\hline & Frequency & Percent & Valid Percent & Cumulative Percent \\
\hline Valid & 1 & 4.5 & 4.5 & 4.5 \\
\cline { 2 - 6 } & 14.00 & 1 & 4.5 & 4.5 & 9.1 \\
\cline { 2 - 6 } & 16.00 & 2 & 9.1 & 9.1 & 18.2 \\
\hline 17.00 & 3 & 13.6 & 13.6 & 31.8 \\
\hline 18.00 & 4 & 18.2 & 18.2 & 50.0 \\
\hline 19.00 & 2 & 9.1 & 9.1 & 59.1 \\
\hline 20.00 & 2 & 9.1 & 9.1 & 68.2 \\
\hline 21.00 & 4 & 18.2 & 18.2 & 96.4 \\
\hline 22.00 & 2 & 9.1 & 9.1 & 100.0 \\
\hline 24.00 & 1 & 4.5 & 4.5 & \\
\hline
\end{tabular}

\section{Pitch Range}

Being monotonous is a prominent symptom in Parkinsonian Dysarthria, it is of utmost importance to investigate both the min pitch (lowest frequency, $\mathrm{Hz}$ ) and max pitch (highest frequency, $\mathrm{Hz}$ ) of a PwP to subsequently being able to draw a singing-based voice rehabilitation plan with good recommendation to repertoires in order to expand pitch range.

Table 7

Descriptive Statistics of Min Pitch

\begin{tabular}{llllllll}
\hline & N & Range & Minimum & Maximum & Mean & $\begin{array}{c}\text { Std. } \\
\text { Deviation }\end{array}$ & Variance \\
\hline Pitchmin & 22 & 191.04 & 138.59 & 329.63 & 214.2741 & 53.18449 & 2828.590 \\
\hline Valid N & 22 & & & & & & \\
(listwise) & & & & & & & \\
\hline
\end{tabular}

Table 7 above shows the descriptive statistics of min pitch among the 22 participants, the lowest score of min pitch was noted at $138.59 \mathrm{~Hz}$ (equivalent to C\#3), whilst the highest score of min pitch was noted at 329.63 (E4), with a total difference in 15 semitones. The mean score of min pitch is noted at $214.2741 \mathrm{~Hz}$ (G\#3), signifying that averagely the lowest note PwP can reach at baseline is at G\#3. 
Table 8

Descriptive Statistics of Max Pitch

\begin{tabular}{lccccccc}
\hline & $\mathrm{N}$ & Range & $\begin{array}{c}\text { Minimu } \\
\mathrm{m}\end{array}$ & $\begin{array}{c}\text { Maximu } \\
\mathrm{m}\end{array}$ & Mean & $\begin{array}{c}\text { Std. } \\
\text { Deviation }\end{array}$ & Variance \\
\hline Pitchmax1 & 22 & 634.61 & 196.00 & 830.61 & 500.8364 & 161.96049 & 26231.19 \\
\hline Valid N & & & & & & & 9 \\
\hline (listwise) & 22 & & & & & & \\
\hline
\end{tabular}

Table 8 above shows the descriptive statistics of max pitch among the 22 participants, the lowest score of max pitch was noted at $196 \mathrm{~Hz}$ (equivalent to G3), whilst the highest score of min pitch was noted at 830.61 (G\#5), with a total difference in 25 semitones. The mean score of max pitch is noted at $500.8364 \mathrm{~Hz}$ (B4), signifying that, in average, the highest note PwP can reach at baseline is at B4.

Table 9

Transcription of Musical Notes to Unit in Frequency $(\mathrm{Hz})$, adapted from Computer Science Department, Aarhus University, $U K$

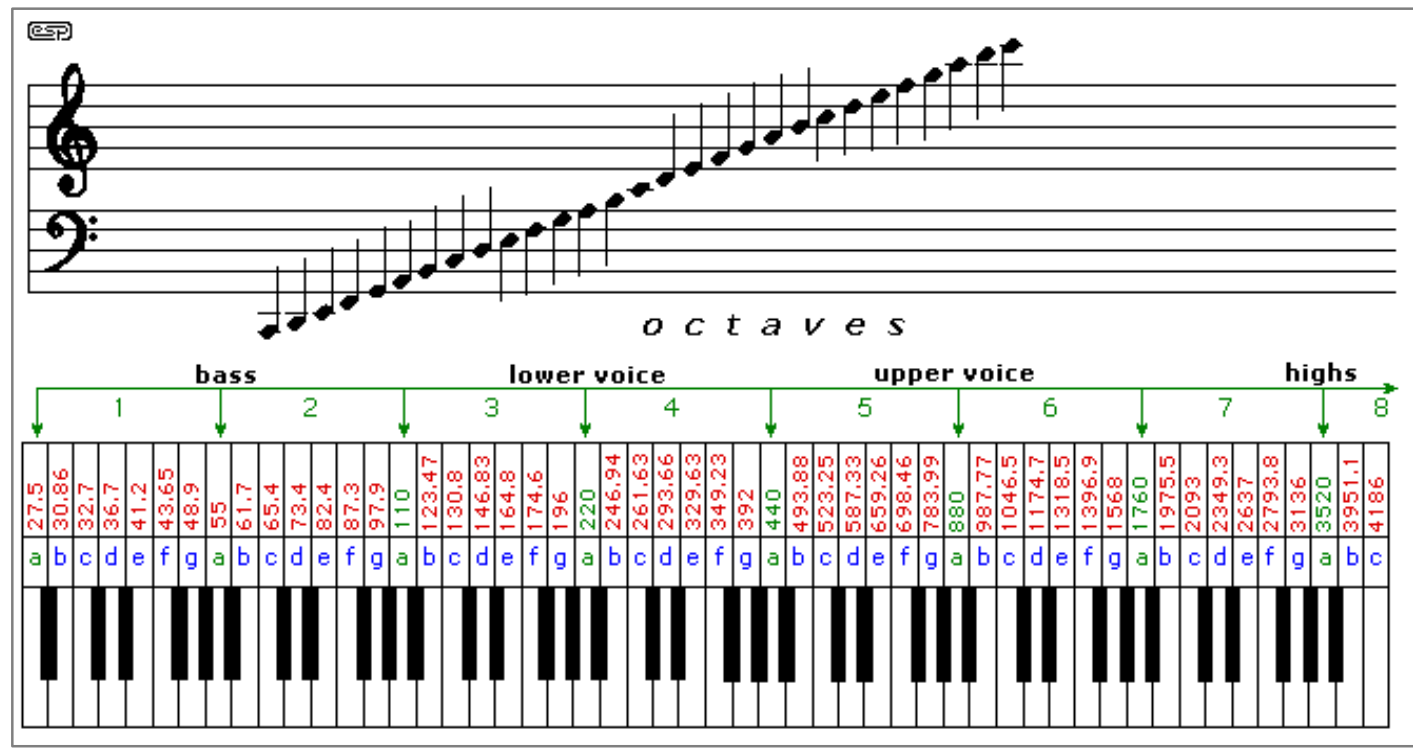

\section{Discussion}

The purpose of this study aimed to explore the baseline vocal parameters among the PwP that include vocal amplitude (loudness), respiratory rate, and pitch range. 
These three parameters were noted as the most prominent symptoms in voice disorders among the PwP, which has seriously affected the effectiveness in delivering voice and/or participate in any daily conversation due to the inaudible volume due to shallow breathing and monotonous pitch.

The findings of the study revealed that mean min vocal amplitude at read was at $52.09 \mathrm{~dB}$, signifying that the softest voice of the PwP in a daily conversation is averagely lower than the normal conversation amplitude standard which is commonly accepted at $60 \mathrm{~dB}$. However, the mean max vocal amplitude at read was noted at $74.68 \mathrm{~dB}$, indicating that the loudest voice of the $\mathrm{PwP}$ in a daily conversation is still audible.

Taking consideration of both min vocal amplitude and max vocal amplitude that should happen normally in a daily conversation following the normal fluctuations of speech patterns, the min vocal amplitude noted in this study revealed that the softest voice in the PwP were below the standard average and posited possibilities of difficulties for listeners to capture all the speech of the PwP due to inconsistency of volume.

On the other hand, the baseline respiratory rate was at the higher side of the healthy range, the mean respiratory rate was noted at 20 breaths/min, showing that the PwP were probably having a higher tendency to develop rapid breathing due to shallow breathing, in another word, the PwP prone to take more breaths than normal in a given time (minute).

Singing activities generally teach an individual to amplify the voice through vocal techniques such as mastering diaphragmatic breathing techniques, training the voice with better vocal resonance via controlling both the consonants and vowels, as well as training the vocal muscles to be in a better closure (Bennett, 2017; Pabon, Stallinga, Södersten, \& Ternström, 2014; Sataloff, 2015). With such, it is hypothesized that with the vocal training through singing, one would be able to have a better control to their vocal and breathing muscles, hence being able to breath deeper to avoid shallow breathing, as well as producing louder voice through better control to the vocal fold's closure.

This study also disclosed that the baseline mean min pitch was $214.2741 \mathrm{~Hz}$ (G\#3), and the baseline mean max pitch was at $500.8364 \mathrm{~Hz}$ (B4). This gives a general yet very helpful overview to music therapists as well as music-based activities' instructors to repertoire planning. A good repertoire plan leads towards successful outcome to expanding pitch ranges; however, a bad repertoire plan could lead to other vocal complications such as more vocal strains, voice damage and laryngeal fatigue.

\section{Conclusion, Implications and Recommendation for Future Research}

The findings of the baseline vocal parameters in the study concluded that the PwP speak in a volume lower than the normally accepted range, they have a tendency to develop rapid breathing due to shallow breathing, and their average pitch range while singing is limited within the range of $\mathrm{G \# 3}-\mathrm{B} 4$. 
These baseline data do not only revealed the current voice problems in the $\mathrm{PwP}$ but also give a clear guideline to music therapists as well as music-based activities' instructors, including voice experts and composers to further planning and/or composing songs that is specifically designed and composed for this cohort, for the purpose of improving voice parameters with vocal training through gradual increment in the level of difficulties of songs in terms of dynamic changes, demands in breathing control with long/short musical phrases and pitch range. Moreover, it also gives the voice activities executives a clearer direction into designing a voice rehabilitative program that better fits into the needs of the PwP.

However, it is recognizable that there were several limitations of this study. First, the study was conducted only among the members in the Malaysian Parkinson's Disease Association where the members are mostly living in Kuala Lumpur or Selangor. Hence, the result in this study is not generalizable to the wider $\mathrm{PwP}$ in Malaysia. Thus, it is recommended that a national scale investigation in the voice characteristics among the PwP should be carried out in order to obtain the data that is more of representative.

Secondly, the parameters that were examined was limited to vocal amplitude, respiratory rate and pitch range, as these were being noted as the most prominent symptoms in voice disorders among the PwP; however, not forgetting that there are also other problems in voice and speech among the PwP such as faded articulation and vocal hoarseness, thus it is important to recommend that more thorough investigation on voice characteristics should be carried out in order to acquire a more comprehensive understanding on the voice problems of this cohort.

Though the result was not able to be generalized to a broad PwP population, and that the parameters being examined were not comprehensive, this study provides an overview in the vocal problems and voice characteristics in the PwP. Music or singing based voice rehabilitation programs for PwP are relatively new in Malaysia, these findings were clearly able to propose an evident direction and draw a clear guideline into shaping a voice rehabilitative program that is of benefit to the $\mathrm{PwP}$ in Malaysia.

\section{References}

Aarsland, D., Larsen, J. P., Karlsen, K., Lim, N. G., \& Tandberg, E. (1999). Mental symptoms in Parkinson's disease are important contributors to caregiver distress. International Journal of Geriatric Psychiatry, 14(10), 866-874. https://doi.org/10.1002/(SICI)1099-1166(199910)14:10<866::AIDGPS38>3.0.CO;2-Z

Atkinson-Clement, C., Sadat, J., \& Pinto, S. (2015). Behavioral treatments for speech in Parkinson's disease: meta-analyses and review of the literature. Neurodegenerative Disease Management, 5(3), 233-248. https://doi.org/10.2217/nmt.15.16

Bennett, G. (2017). The science of singing : A voice lesson from anatomy and physiology (pp. 0-15). Cedarville University. Retrieved from https://digitalcommons.cedarville.edu/research_scholarship_symposium/2017/ 
podium_presentations/1/

Branchi, I., D’Andrea, I., Armida, M., Cassano, T., Pezzola, A., Potenza, R. L., Morgese, M. G, Popoli, P., Alleva, E. (2008). Nonmotor symptoms in Parkinson's disease: Investigating early-phase onset of behavioral dysfunction in the 6-hydroxydopamine-lesioned rat model. Journal of Neuroscience Research, 86(9), 2050-2061. https://doi.org/10.1002/jnr.21642

Buetow, S. A., Talmage, A., McCann, C., Fogg, L., \& Purdy, S. (2014). Conceptualizing how group singing may enhance quality of life with Parkinson's disease. Disability and Rehabilitation, 36(5), 430-433. https://doi.org/10.3109/09638288.2013.793749

Cacciatore, F. M., Simone, D. De, Patrocinio, D., \& Sallustio, V. (2012). Adverse drug reaction to dopamine agonists (DA) or appearance of a typical sign of Parkinson's Disease induced by DA. Basal Ganglia, 2(3), e1-e87. https://doi.org/10.1016/j.baga.2012.10.001

Clark, J. P., Adams, S. G., Dykstra, A. D., Moodie, S., \& Jog, M. (2014). Loudness perception and speech intensity control in Parkinson's disease. Journal of Communication Disorders, 51, 1-12. https://doi.org/10.1016/j.jcomdis.2014.08.001

Dyduch, A., \& Załuska, M. (2014). Depression in Parkinson's disease: the effectiveness and risk of pharmacotherapy. Clinical review. Psychogeriatrics: The Official Journal of the Japanese Psychogeriatric Society, 1-7. https://doi.org/10.1111/psyg.12078

Elefant, C., Baker, F. a, Lotan, M., Lagesen, S. K., \& Skeie, G. O. (2012). The Effect of Group Music Therapy on Mood, Speech, and Singing in Individuals with Parkinson's Disease -- A Feasibility Study. Journal of Music Therapy, 49(3), 278-302. https://doi.org/10.1093/jmt/49.3.278

Elefant, C., Lotan, M., Baker, F. a., \& Skeie, G. O. (2012). Effects of music therapy on facial expression of individuals with Parkinson's disease: A pilot study. Musicae Scientiae, 16(3), 392-400. https://doi.org/10.1177/1029864912458917

Evans, C., Canavan, M., Foy, C., Langford, R., \& Proctor, R. (2012). Can group singing provide effective speech therapy for people with Parkinson's disease? Arts \& Health, 4(1), 83-95. https://doi.org/10.1080/17533015.2011.584883

Fogg-Rogers, L., Buetow, S., Talmage, A., McCann, C. M., Leao, S. H. S., Tippett, L., Leung, J., McPherson, K. M., Purdy, S. C. (2015). Choral singing therapy following stroke or Parkinson's disease: An exploration of participants' experiences. Disability and Rehabilitation, 8288(November), 1-11. https://doi.org/10.3109/09638288.2015.1068875

Haneishi, E. (2001). Effects of a music therapy voice protocol on speech intelligibility, vocal acoustic measures, and mood of individuals with Parkinson's disease. Journal of Music Therapy, 38(4), 273-290. https://doi.org/10.1093/jmt/38.4.273

Jankovic, J. (2008). Parkinson's disease: Clinical features and diagnosis. Journal of Neurology, Neurosurgery \& Psychiatry, 79(4), 368-376. https://doi.org/10.1136/jnnp.2007.131045

Jenkinson, C., Peto, V., Fitzpatrick, R., Greenhall, R., Hyman, N. (1995) Self- 
reported Functioning and Well-being in Patients with Parkinson's Disease: Comparison of the Short-form Health Survey (SF-36) and the Parkinson's Disease Questionnaire (PDQ-39). Age and Ageing, 24, 505-509.

Lim, S.-Y., Fox, S. H., \& Lang, A. E. (2009). Overview of the extranigral aspects of Parkinson disease. Archives of Neurology, 66(2), 167-172. https://doi.org/10.1001/archneurol.2008.561

Lim, S.-Y., Puvanarajah, S., Lee, M. K., Chee, K. Y., Viswanathan, S., Tan, A. H., Chong, K. T., Azrin, K. (2012). 2012 Consensus Guidelines for the Treatment of Parkinson's Disease.

Meireles, J., \& Massano, J. (2012). Cognitive impairment and dementia in Parkinson's disease: Clinical features, diagnosis, and management. Frontiers in Neurology, MAY(May), 1-16. https://doi.org/10.3389/fneur.2012.00088

Miller, N., Deane, K. H. O., Jones, D., Noble, E., \& Gibb, C. (2011). National survey of speech and language therapy provision for people with Parkinson's disease in the United Kingdom: Therapists' practices. International Journal of Language \& Communication Disorders / Royal College of Speech \& Language Therapists, 46(2), 189-201. https://doi.org/10.3109/13682822.2010.484849

Miller, N., Noble, E., Jones, D., \& Burn, D. (2006). Life with communication changes in Parkinson's disease. Age and Ageing, 35(3), 235-239. https://doi.org/10.1093/ageing/afj053

Pabon, P., Stallinga, R., Södersten, M., \& Ternström, S. (2014). Effects on vocal range and voice quality of singing voice training: The classically trained female voice. Journal of Voice, 28(1), 36-51. https://doi.org/10.1016/j.jvoice.2013.06.005

Pacchetti, C., Mancini, F., Aglieri, R., Fundarò, C., Martignoni, E., \& Nappi, G. (2000). Active music therapy in Parkinson's disease: An integrative method for motor and emotional rehabilitation. Psychosomatic Medicine, 62, 386-393. https://doi.org/10.1097/00006842-200005000-00012

Pagonabarraga, J., Kulisevsky, J., Strafella, A. P., \& Krack, P. (2015). Apathy in Parkinson's disease: Clinical features, neural substrates, diagnosis, and treatment. The Lancet Neurology, 14(5), 518-531. https://doi.org/10.1016/S1474-4422(15)00019-8

Pinto, S., Ozsancak, C., Tripoliti, E., Thobois, S., Limousin-dowsey, P., \& Auzou, P. (2004). Review treatments for dysarthria in Parkinson's disease. Neurology, 3(September), 547-556.

Politis, M., Wu, K., Molloy, S., Bain, P. G., Chaudhuri, K. R., \& Piccini, P. (2010). Parkinson's disease symptoms: The patient's perspective. Movement Disorders, 25(11), 1646-1651. https://doi.org/10.1002/mds.23135

Ramig, L., \& Fox, C. (2007). Speech disorders in Parkinson's disease and the effects of pharmacological, surgical and speech treatment with emphasis on Lee Silverman voice treatment (LSVT). Parkinson's Disease Foundation News and Review.

Ramig, L. O., Fox, C., \& Sapir, S. (2007). Speech disorders in Parkinson's disease and the effects of pharmacological, surgical and speech treatment with emphasis on Lee Silverman voice treatment (LSVT). Handbook of Clinical 
Neurology (Vol. 83, pp. 385-399). https://doi.org/10.1016/S00729752(07)83017-X

Richardson, K., Sussman, J. E., Stathopoulos, E. T., \& Huber, J. E. (2014). The effect of increased vocal intensity on interarticulator timing in speakers with Parkinson's disease: A preliminary analysis. Journal of Communication Disorders, 52, 44-64. https://doi.org/10.1016/j.jcomdis.2014.09.004

Rutten, S., Ghielen, I., Vriend, C., Hoogendoorn, A. W., Berendse, H. W., Leentjens, A. F. G., ... van den Heuvel, O. A. (2014). Anxiety in Parkinson's disease: Symptom dimensions and overlap with depression and autonomic failure. Parkinsonism and Related Disorders. https://doi.org/10.1016/j.parkreldis.2014.11.019

Sataloff, R. T. (2015). Book Review: Singing and Science: Body, Brain and Voice. Journal of Voice, 29(1), e1. https://doi.org/10.1016/j.jvoice.2014.06.010

Schulz, G. M., \& Grant, M. K. (2000). Effects of speech therapy and pharmacologic and surgical treatments on voice and speech in Parkinson's disease: A review of the literature. Journal of Communication Disorders, 33, 59-88. https://doi.org/10.1016/S0021-9924(99)00025-8

Schulz, G. M., Greer, M., \& Friedman, W. (2000). Changes in vocal intensity in Parkinson's disease following pallidotomy surgery. Journal of Voice:Official Journal of the Voice Foundation, 14(4), 589-606. https://doi.org/10.1016/S0892-1997(00)80015-6

Scott, S., \& Caird, F. I. (1983). Speech therapy for Parkinson' s disease. Journal of Neurology, Neurosurgery, and Psychiatry, 46(October 1982), 140-144. Retrieved from http://jnnp.bmj.com/content/46/2/140.full.pdf+html

Tanday, S. (2016). Feature How the power of singing is helping patients to breathe again. The Lancet Respiratory, 2600(16), 2600. https://doi.org/10.1016/S22132600(16)00002-3

Wertheimer, J., Gottuso, A. Y., Nuno, M., Walton, C., Duboille, A., Tuchman, M., \& Ramig, L. (2014). The impact of STN deep brain stimulation on speech in individuals with Parkinson's disease: The patient's perspective. Parkinsonism and Related Disorders, 20(10), 1065-1070.

https://doi.org/10.1016/j.parkreldis.2014.06.010

\section{Biography}

Mei Foong is a soprano and an opera singer. She graduated with a Bachelor of Fine Arts from National Taiwan Normal University (NTNU, Taiwan). She furthered her vocal studies at Conservatorio di Musica Santa Cecilia (Rome, Italy) and subsequently gained her master's degree at the University of Melbourne (Australia). She is now a PhD candidate at University Putra Malaysia (UPM), in which her present research focuses on the effects of singing for voice rehabilitation on people with Parkinson's Disease (PwP). She has given several research presentations on voice rehabilitations on $\mathrm{PwP}$ at a few international conferences. Earlier this year, she was awarded and recognised as the Goodwill Ambassador for the Malaysian Parkinson's Disease Association (MPDA). 